\title{
不同密度旱柳的树冠构型与光截获
}

\author{
陈 静 赵成章 ${ }^{*}$ 王继伟 赵连春 \\ 西北师范大学地理与环境科学学院, 甘肃省湿地资源保护与产业发展工程研究中心, 兰州 730070
}

\begin{abstract}
摘 要 植物可以通过调整树冠构型和光截获来增强自身的光合效率和竞争力。在甘肃省张掖市黑河干流边缘的洪泛平原湿 地选取I (25-36 Ind. plot $^{-1}$ )、II (37-48 Ind. plot ${ }^{-1}$ )和III (49-60 Ind. plot $^{-1}$ ) 3个密度梯度, 研究了不同密度旱柳(Salix matsudana) 的冠层厚度、冠层面积和冠层光截获, 以及它们的相互关系。结果表明: 随旱柳种群密度增加, 土壤水分逐渐增加、土壤电 导率和水分利用率逐渐减小, 旱柳枝长度与冠层厚度呈逐渐增大的趋势, 光合有效辐射、分枝数、分枝角度与冠层面积呈逐 渐减小的趋势, 叶面积指数和光截获、净光合速率和蒸腾速率在中密度样地最大; 旱柳的光截获与冠层厚度和冠层面积在低 密度样地分别呈极显著正相关和负相关关系, 在高密度样地呈显著正相关和负相关关系 $(p<0.05)$, 在中密度样地均呈极显著 正相关关系 $(p<0.01)$ 。旱柳在低密度选择减小光截获与冠层厚度而增大冠层面积的水平空间拓展模式, 在高密度倾向于垂直 空间拓展模式, 反映了植物树冠构型在不同生境中的表型可塑性变化。
\end{abstract}

关键词 光截获; 冠层厚度; 冠层面积; 密度; 旱柳

引用格式: 陈静, 赵成章, 王继伟, 赵连春 (2017). 不同密度旱柳的树冠构型与光截获. 植物生态学报, 41, 661-669. doi: 10.17521/cjpe.2016.0257

\section{Canopy structure and radiation interception of Salix matsudana: Stand density dependent re- lationships}

CHEN Jing, ZHAO Cheng-Zhang ${ }^{*}$, WANG Ji-Wei, and ZHAO Lian-Chun

College of Geography and Environmental Science, Northwest Normal University, Research Center of Wetland Resources Protection and Industrial Development Engineering of Gansu Province, Lanzhou 730070, China

\section{Abstract}

Aims Plants can enhance their photosynthetic efficiency and competitiveness by adjusting canopy structure and radiation interception. The objective of this paper was to quantify the relationship between canopy structure (crown depth and crown area) and light interception $(L I)$ in a Salix matsudana stand under three different stand densities in a flood plain of Zhangye.

Methods Our study site is located at the Heihe flood plain of Xichengyi in Ganzhou district, Zhangye City, Gansu Province in the middle Heihe River, where S. matsudana is the dominant species. Based on stand density

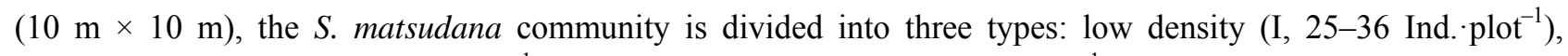
medium density (II, 37-48 Ind. plot $^{-1}$ ), and high density (III, 49-60 Ind. plot $^{-1}$ ). Community characteristics, soil physical and chemical properties of each type were measured. At each plot, we measured photosynthetically active radiation $(P A R), L I$, net photosynthetic rate $\left(P_{\mathrm{n}}\right)$, transpiration rate $\left(T_{\mathrm{r}}\right)$, crown depth, crown area, leaf area index $(L A I)$, twig numbers, twig length, and bifurcation angle. The standardized major axis (SMA) estimation method was used to determine the relationships between $L I$ and canopy structure.

Important findings With increasing in stand density, we found that soil moisture increased, and soil electric conductivity decreased, while twig length and crown depth increased, and PAR, twig numbers, bifurcation angle and crown area decreased. $L A I$ and $L I, P_{\mathrm{n}}$ and $T_{\mathrm{r}}$ reached their maximum at the stand of medium density. There was a significant, positive correlation and negative correlation $(p<0.01)$, respectively, between the $L I$, crown depth and crown area at low density (I), whereas low significant $(p<0.05)$ at high density (III), and high significantly positive correlation $(p<0.01)$ at the medium density (II). S. matsudana has more horizontal branches that reduce LI. Canopy thickness and increased crown area at low density. More vertical distribution of branches at high density, and a more balanced spacial distribution were found at medium density.

Key words light interception; crown depth; crown area; density; Salix matsudana

收稿日期Received: 2016-08-04 接受日期Accepted: 2017-05-03

* 通信作者Author for correspondence (E-mail: zhaocz601@163.com) 
Citation: Chen J, Zhao CZ, Wang JW, Zhao LC (2017). Canopy structure and radiation interception of Salix matsudana: Stand density dependent relationships. Chinese Journal of Plant Ecology, 41, 661-669. doi: 10.17521/cjpe.2016.0257

森林冠层结构决定了光能在冠层内的传输和 分布方式(Goel, 1988), 对植物利用生态空间的能力 和提高光合收益具有重要意义。光截获 $(L I)$ 和冠层 形态结构能够反映植物冠层适应特定生境的基本行 为和光合生理功能, 体现了植物进行资源优化配置 的生态适应与生存策略(Poorter et al., 2003)。LI作为 冠层内部光分布特征的一个评价指标, 反映了树冠 对太阳光的截取能力(Wunsche et al., 1996), 是度量 群体内光照状况及光捕获能力的重要参数(李世莹 等, 2013); 树冠构型可以描述为垂直和水平截面上 的形状, 其中冠层厚度或冠长表示树冠垂直空间大 小，表征树冠的纵向生长与邻体间的竞争效应 (Thorpe et al., 2010), 树冠面积是研究树冠水平伸 展空间结构的指标, 反映植物在水平方向上的光资 源竞争能力(Kind et al., 2006)。植物可以通过调整冠 层厚度和面积来适应生境变化, $L I$ 与冠层构型相互 影响、相互制约的生态学机制是理解冠层内光合作 用过程和光合碳固定的重要依据。

密度是自然界植物种群普遍存在的重要选择 压力(Japhet et al., 2009), 随着种群密度的增加, 林 冠内光量减少, 邻株的物理或几何上的拥挤效应会 加剧种内资源竞争, 影响到植物的光资源获取能力, 植物为获取最大光资源量, 在垂直生长与侧向扩展 间物质营养投入的权衡发生偏移, 引起构型转变 (Clark, 2010), 促使植物对叶面积指数 ( $L A I)$ 、分枝角 度、分枝数和枝长度进行调整, 构建与光截获效率 相适应的冠幅厚度与冠幅面积, 体现了最大化地吸 取异质性分布资源的受食行为和可塑性反应。因此, 研究不同密度下林冠 $L I$ 和冠层构型的关系, 对于理 解植物的光截获对策具有重要意义。

旱柳(Salix matsudana)是中国北方主要道路防 护林及沙荒造林树种, 抗寒喜光、生长快、繁殖容 易, 具有较好的防风固沙和保持水土功能, 在改善 生态环境中发挥了重要作用。近年来, 国内外学者 关于冠层光照与冠层构型的关系(Poorter et al., 2008; 杨晓东等, 2013)、湿地环境因子对植物光合 和形态的影响(Rodríguez-González et al., 2010)、不 同密度冠层构型的生态生理响应机制(章建红等, 2014)以及旱柳的光合效率(夏江宝等, 2013)、生长潜 力(Sixto et al., 2015)和生理特性(Song et al., 2016)等
方面有较多研究, 解释了植物适应特定环境的光 照、光合生理、形态变化与表型塑造, 但光分布和 旱柳冠层构型的关联性研究不够深入, 特别是在湿 地群落不同密度生境中, $L I$ 和冠层厚度、冠层面积关 系对植物生理功能和异质性资源配置的响应机理尚 不清晰。鉴于此, 本研究以黑河中游张掖段洪泛平 原湿地的天然旱柳林为研究对象, 研究旱柳在密度 梯度上 $L I$ 与冠层厚度和面积的关系, 旨在揭示湿地 植物密度制约下光分布规律与表型可塑性。

\section{1 材料和方法}

\section{1 研究区概况}

研究区位于甘肃省黑河流域中游张掖市甘州区 西城驿的黑河滩地, 地理位置为 $38.98^{\circ} \mathrm{N}, 100.40^{\circ} \mathrm{E}$, 海拔 $1482.7 \mathrm{~m}$, 属温带大陆性气候, 年平均气温 $7.8{ }^{\circ} \mathrm{C}, \geqslant 0{ }^{\circ} \mathrm{C}$ 积温为 $2734{ }^{\circ} \mathrm{C}$, 年降水量 $132.6 \mathrm{~mm}$, 主要集中在6-9月, 年蒸发量1 $986.5 \mathrm{~mm}$, 年日照时 间 $3077 \mathrm{~h}$ (韩玲等, 2016)。土壤以砾石土、灰棕荒漠 土和草甸土为主，植被分布具有明显的水平分异特 征, 以湿生植物、盐生植物与陆生乔灌林木为主。 主要植物有: 旱柳、宽苍水柏枝(Myricaria bracteata)、多枝柽柳(Tamarix ramosissima)、沙菄(Elaeagnus angustifolia)、苦豆子(Sophora alopecuroides)、 芨芨草(Achnatherum splendens)、冰草(Agropyron cristatum)、芦苇(Phragmites australis)、节节草(Equisetum ramosissimum)等(韩玲等, 2016)。该区属于 张掖黑河国家湿地核心区，没有人类活动的影响， 较为真实地反映了湿地的自然属性与植物生物学特 性的关系。

\section{2 样地设置与调查方法}

本次试验地选择在黑河河床汊沟交织处的河 心滩边稍高的、有旱柳广泛分布的洪泛平原。于2015 年8月上旬, 根据实地考察, 选择一块发育阶段相对 一致的旱柳林湿地种群, 布置长 $200 \mathrm{~m}$ 、宽 $50 \mathrm{~m}$ 的水 平样带 1 条, 整个样带划分为 100 个 $10 \mathrm{~m} \times 10 \mathrm{~m}$ 的样 方, 以“株”为取样单位进行实验, 样方内胸径大于 $1 \mathrm{~cm}$ 的立木个体数的变化区间为 $25-60$ 株。由于研究 对象为天然林, 无法人为控制密度区组, 我们按照 旱柳种群密度的变异范围, 根据等距离的原则(章 建红等, 2014), 以12为间隔等分, 将所有样方按低密 
度(I, 25-36 Ind. $\cdot$ plot $\left.^{-1}\right)$ 、中密度(II, 37-48 Ind. $\cdot$ plot $^{-1}$ ) 和高密度(III, 49-60 Ind. plot $^{-1}$ )划分为 3 组, 样方数 分别为 $26 、 38$ 和 36 个, 选取所有样方。用手持GPS 仪测量每个样方的经纬度和海拔, 对各样方内胸径 $\geqslant 1 \mathrm{~cm}$ 的每木个体进行群落特征调查, 记录株高、 胸径、郁闭度。

\section{3 测试项目和方法}

\subsection{1 土壤取样与理化性质测量}

(1)土壤含水量采样。土壤取样于 2015 年 8 月下 旬完成, 取样时间内基本无明显降水, 土壤水盐状 况相对稳定。在每个小样方随机选取 3 个样点挖掘 $1 \mathrm{~m} \times 1 \mathrm{~m} \times 0.5 \mathrm{~m}$ 土壤剖面, 用环刀分5层间隔 $10 \mathrm{~cm}$ 采取土样, 现场编号、称鲜质量后带回实验室 在 $105{ }^{\circ} \mathrm{C}$ 的烘箱内烘 $12 \mathrm{~h}$, 取出称质量, 采用公式: 土壤质量含水量 $(\%)=$ (湿土质量-烘干土质量) / 烘干 土质量, 计算出各样地 0-50 cm 土层土壤质量含水 量(韩玲等, 2016)。

(2)土壤盐分采样。用电导法测定土壤含盐量。 在室温下称取过 $2 \mathrm{~mm}$ 篎的风干土样 $10 \mathrm{~g}$, 加 $50 \mathrm{~mL}$ 去 $\mathrm{CO}_{2}$ 蒸馏水 (水土质量比为 $5: 1$ ), 取浸出液, 置振 荡机上振荡 $5 \mathrm{~min}$ 。将布氏漏斗与抽气系统相连后把 悬浊的土浆缓缓倒入漏斗, 直至抽滤完毕, 滤液倒 入三角瓶备用(张雅琼等, 2010)。用上海雷磁仪器厂 生产的DDS-11C便携式电导仪测浸出液的电导率, 3 次重复, 取平均值。

\subsection{2 光合有效辐射和光截获测定}

选择晴朗无云的天气用SUNSCAN冠层分析仪 (Delta T, Cambridge, UK)测定每个样方的光合有效 辐射 $(P A R)$ 与 $L I$ 。测定位置分别在旱柳的上方(距树 顶 $50 \mathrm{~cm}$ )、中层(株高1/2处)和地表(距地表 $5 \mathrm{~cm}$ 处), 每个样方的不同位置测定 3 次。倘若测定位置在树冠 中层和上方, 测定时需要架设铝合金人字梯。 $L I$ 按 公式 $L I=\left(I_{0}-I\right) / I_{0}$ 进行计算, 式中, $I_{0}$ 为冠层顶部瞬 时 $P A R ; I$ 为通过一定叶层后的瞬时 $P A R$ 。

\subsection{3 植物光合参数测定。}

选择晴朗天气的9:00-12:00, 在每个样方选取 大中小各两株旱柳作为供试植株, 每株选取最外层 4 个方位充分伸展且健康完整的2-3个叶片, 利用 GFS-3000便携式光合测量系统(Heinz Walz GmbH, Bavaria, Germany)进行每个标记叶片光合参数的 测定。测量过程中使用人工红蓝光源, $P A R$ 定为 $1200 \mu \mathrm{mol} \cdot \mathrm{m}^{-2} \cdot \mathrm{s}^{-1}, \mathrm{CO}_{2}$ 浓度约为 $340 \mu \mathrm{mol} \cdot \mathrm{mol}^{-1}$,
相对湿度 $(R H)$ 保持在 $40 \%-50 \%$, 流速设定为 $750 \mu \mathrm{mol} \cdot \mathrm{s}^{-1}$, 叶室面积为 $8 \mathrm{~cm}^{2}$, 每个叶片记录 5 组 数据用于统计分析, 分别测定叶片的净光合速率 $\left(P_{\mathrm{n}}\right)$ 、蒸腾速率 $\left(T_{\mathrm{r}}\right)$ 等参数, 测试后的叶片保存于湿 润的封口袋中, 带回实验室, 用便携式激光叶面积 仪(CI-202, CID, Camas, USA)测定叶面积。水分利 用率 $(W U E)=P_{\mathrm{n}} \times T_{\mathrm{r}}^{-1}$ 。

\subsection{4 冠层构型测量}

(1)冠层厚度和冠层面积。测定每个样方内胸径 $>1 \mathrm{~cm}$ 的所有个体, 冠幅长度依个体的垂直投影面 积直接用皮尺测定; 叶下高和树高用测高仪 (Vertex-IV, Haglof, Dalarna, Sweden)测定。冠层厚度 与冠层面积采用以下公式计算:

$$
\begin{aligned}
& C D=H_{\mathrm{H}}-H_{\mathrm{LH}} \\
& C A=X_{\mathrm{EW}} \times Y_{\mathrm{SN}}
\end{aligned}
$$

式中, $C D$ 为冠层厚度, $H_{\mathrm{H}}$ 表示树高, $H_{\mathrm{LH}}$ 表示叶下高; $C A$ 表示冠层面积, $X_{\mathrm{EW}}$ 为树冠垂直投影在东西方向 上的距离, $Y_{\mathrm{SN}}$ 为树冠垂直投影在南北方向上的距离 (杨晓东等, 2013)。

(2) 叶面积指数 $(L A I)$ 。采用CI-110数字式植物冠 层结构分析仪(CI-110, CID, Camas, USA)测定每个 样方的冠层结构及光辐射指标。于早晚没有强烈直 射光时, 将安装有鱼眼探测头的观测棒分别定点在 样地中心、中线和对角线 $1 / 4$ 处, 共 9 个测定点进行测 量, 每块样地拍摄9幅照片(马泽清等, 2008)。通过CI110 计算机图像数字化专用软件分析后, 可得到 $L A I$ 。

(3)小枝构型。对每块样地内的所有旱柳进行编 号, 按编号顺序记录每株旱柳的分枝数。在每株旱 柳东西南北 4 个方位各选择 1 个枝条(枝条数量 $\leqslant 4$ 则全部选择), 对于选择的每个大枝, 定义最外层的 当年生枝为第 1 层级枝条, 向内第 2 层级为支撑枝, 并标记 1 个无明显损失的小枝, 在现场用量角器测 量标记的当年生小枝与其支撑枝之间的夹角, 然后 将标记的小枝剪取并放入塑料袋, 编码后带回实验 室用直尺测量小枝长度(杜晶等, 2016)。

\section{4 数据分析}

依据密度将 100 个样方划分为 3 个密度组(分别 为I、II、III), 各组的样方数和旱柳种群特征见表 1 。 对旱柳种群样地划分出的 3 个密度梯度各平均性状 进行统计分析。旱柳光截获、冠层厚度和冠层面积 的实验数据先进行以 10 为底的对数转换, 使之符合 正态分布后再进行分析。数据分析主要运用标准化 
表1 样地分组情况和旱柳种群的生物学特征(平均值土标准误差)

Table 1 Biological characteristics of Salix matsudana population (mean $\pm S E$ ) at three stands of variable density

\begin{tabular}{|c|c|c|c|c|c|}
\hline $\begin{array}{l}\text { 密度分组 } \\
\text { Density fractions }\end{array}$ & $\begin{array}{c}\text { 密度区间 } \\
\text { Density interval (Ind. } \cdot \operatorname{plot}^{-1} \text { ) }\end{array}$ & $\begin{array}{c}\text { 样方数 } \\
\text { Number of plots }\end{array}$ & $\begin{array}{c}\text { 高度 } \\
\text { Height }(\mathrm{cm})\end{array}$ & $\begin{array}{c}\text { 胸径 } \\
\text { Diameter at breast height }(\mathrm{cm})\end{array}$ & $\begin{array}{c}\text { 郁闭度 } \\
\text { Crown density }(\%)\end{array}$ \\
\hline 低密度 Low density (I) & $25-36$ & 26 & $398.57 \pm 20.63^{c}$ & $13.06 \pm 0.61^{\mathrm{a}}$ & $42.84 \pm 2.72^{\mathrm{c}}$ \\
\hline 中密度 Medium density (II) & $37-48$ & 38 & $454.69 \pm 24.05^{\mathrm{b}}$ & $10.72 \pm 0.58^{\mathrm{b}}$ & $69.56 \pm 3.93^{\mathrm{b}}$ \\
\hline 高密度 High density (III) & $49-60$ & 36 & $521.23 \pm 27.48^{\mathrm{a}}$ & $8.64 \pm 0.39^{\mathrm{c}}$ & $87.09 \pm 4.37^{\mathrm{a}}$ \\
\hline
\end{tabular}

同列不同小写字母表示密度间差异显著 $(p<0.01)$ 。

Different lowercase letters in the same column indicate significant difference among density at $p<0.01$ level.

主轴估计(SMA)的方法(Warton et al., 2006)对旱柳 光截获和冠层构型间的关系进行分析, 采用Excel 软件和SPSS 16.0 统计分析软件对数据进行相关分 析和方差分析, 显著性水平设为 0.05 , 方差分析前 对所有数据进行方差齐性检验和正态分布检验。

\section{2 结果和分析}

\section{1 不同密度旱柳群落土壤性质与光合性状}

不同密度旱柳群落土壤理化性质与光合特性 的变化如表2所示。旱柳土壤电导率、PAR、LI、 $P_{\mathrm{n}}$ 、 $T_{\mathrm{r}}$ 和WUE在不同密度存在显著差异 $(p<0.05)$, 随种 群密度的增加, 土壤电导率、 $P A R$ 和水分利用率呈逐 渐减小趋势，分别降低了 $69.55 \%$ 、55.70\%和 $24.36 \%$; $L I 、 P_{\mathrm{n}}$ 和 $T_{\mathrm{r}}$ 呈倒U形变化趋势: $L I$ 和 $T_{\mathrm{r}}$ 表现为 $\mathrm{II}>\mathrm{III}>\mathrm{I}$, 增幅分别达到 $26.56 \%$ 和 $42.05 \%$, 而 $P_{\mathrm{n}}$ 为 II $>$ I $>$ III, 增幅达到 $47.84 \%$; 土壤质量含水量在不同密度均出 现较大值, 无显著差异 $(p>0.05)$ 。

\section{2 不同密度旱柳冠层构型的变化}

如图1所示, 旱柳冠层构型在不同密度的变化 存在显著差异 $(p<0.05)$ 。随着密度的逐渐增加, 旱 柳的冠层厚度与枝长度呈逐渐增加趋势, 从密度 I 到密度III分别增加了 $66.67 \%$ 和 $44.80 \%$; 冠层面积、 分枝数和分枝角度呈逐渐减小趋势, 密度 $\mathrm{I}$ 到密度 III分别减小 $24.69 \% 、 60.89 \%$ 和 $28.33 \%$; LAI呈先增大
后减小的趋势, 变化趋势为 $I I>I I I>I$, 即在密度 $I I$ 出现最大值, 密度I出现最小值, 密度II的LAI较密度 I与密度III分别增大了 $132.39 \%$ 和 $23.13 \%$ 。

\section{3 不同密度旱柳冠层光截获与构型的关系}

\subsection{1 光截获与冠层厚度的关系}

如图2所示, 旱柳冠层 $L I$ 与冠层厚度在低密度 $\mathrm{I}$ 和中密度II呈极显著正相关关系 $(p<0.01$, 图2I、图 $2 \mathrm{II})$, 高密度III呈显著正相关关系 $(p<0.05$, 图 $2 \mathrm{III})$ 。 通过SMA分析得出 3 个不同密度梯度间的斜率无显 著差异 $(p>0.05), L I$ 与冠层厚度回归关系具有共同 斜率 $0.74(95 \%$ 的置信区间 $C I=(0.67,0.83))$, 显著小 于1 $(p<0.05)$, 表明 LI增加比率大于冠层厚度的增 加比率。随着种群密度逐渐增加, $L I$ 与冠层厚度的 $y$ 轴截距显著下降 $(p<0.05)$, 表明相同的 $L I$ 条件下, 种群密度越低, 冠层厚度越小。

\subsection{2 光截获与冠层面积的关系}

如图3所示, 旱柳冠层 $L I$ 与冠层面积在低密度 $\mathrm{I}$ 和高密度III分别呈极显著负相关 $(p<0.01$, 图3I)与显 著负相关 $(p<0.05$, 图3III), 中密度II则呈极显著正 相关关系 $(p<0.01$, 图3II); 随着种群密度逐渐增加, 旱柳 $L I$ 和冠层面积回归方程的 $\mathrm{SMA}$ 斜率分别为 $-0.55(95 \%$ 的置信区间 $C I=(-0.72,-0.39)) 、 0.52$ $(95 \%$ 的置信区间 $C I=(0.43,0.62)) 、-0.63(95 \%$ 的置 信区间 $C I=(-0.78,-0.45))$, 低密度和高密度旱柳的

表2 不同密度旱柳群落土壤性质与光合性状(平均值土标准误差)

Table 2 Soil characteristics and photosynthetic characteristics of Salix matsudana stand by density (mean $\pm S E)$

\begin{tabular}{|c|c|c|c|}
\hline & \multicolumn{3}{|c|}{ 密度 Density } \\
\hline & I & II & III \\
\hline 土壤质量含水量 Mass moisture of soil (\%) & $31.56 \pm 1.52^{\mathrm{a}}$ & $34.83 \pm 1.87^{\mathrm{a}}$ & $38.47 \pm 2.01^{\mathrm{a}}$ \\
\hline 土壤电导率 Soil electric conductivity $\left(\mathrm{ms} \cdot \mathrm{cm}^{-1}\right)$ & $7.75 \pm 0.41^{\mathrm{a}}$ & $4.09 \pm 0.21^{\mathrm{b}}$ & $2.36 \pm 0.13^{\mathrm{c}}$ \\
\hline 光合有效辐射 $P A R\left(\mu \mathrm{mol} \cdot \mathrm{m}^{-2} \cdot \mathrm{s}^{-1}\right)$ & $981.26 \pm 50.14^{\mathrm{a}}$ & $740.51 \pm 39.08^{b}$ & $434.67 \pm 24.34^{\mathrm{c}}$ \\
\hline 光截获 $L I$ & $0.64 \pm 0.03^{\mathrm{c}}$ & $0.81 \pm 0.05^{\mathrm{a}}$ & $0.73 \pm 0.04^{\mathrm{b}}$ \\
\hline 净光合速率 $P_{\mathrm{n}}\left(\mu \mathrm{mol} \cdot \mathrm{m}^{-2} \cdot \mathrm{s}^{-1}\right)$ & $10.27 \pm 0.53^{b}$ & $13.69 \pm 0.61^{\mathrm{a}}$ & $9.26 \pm 0.47^{\mathrm{c}}$ \\
\hline 蒸腾速率 $T_{\mathrm{r}}\left(\mathrm{mmol} \cdot \mathrm{m}^{-2} \cdot \mathrm{s}^{-1}\right)$ & $4.59 \pm 0.23^{\mathrm{c}}$ & $6.52 \pm 0.34^{\mathrm{a}}$ & $5.24 \pm 0.27^{\mathrm{b}}$ \\
\hline 水分利用率 $W U E\left(\mu \mathrm{mol} \cdot \mathrm{mmol}^{-1}\right)$ & $2.34 \pm 0.11^{\mathrm{a}}$ & $2.10 \pm 0.09^{\mathrm{b}}$ & $1.77 \pm 0.08^{\mathrm{c}}$ \\
\hline
\end{tabular}

同行不同小写字母表示密度间差异显著 $(p<0.05)$ 。I、II、III, 同表 1 。

$P A R$, photosynthetically active radiation; $L I$, light interception; $P_{\mathrm{n}}$, net photosynthetic rate; $T_{\mathrm{r}}$, transpiration rate; WUE, water use efficiency. Different lowercase letters in the same line indicate significant differences of different density for identical module $(p<0.05)$. I, II, III, see Table 1 .

www.plant-ecology.com 

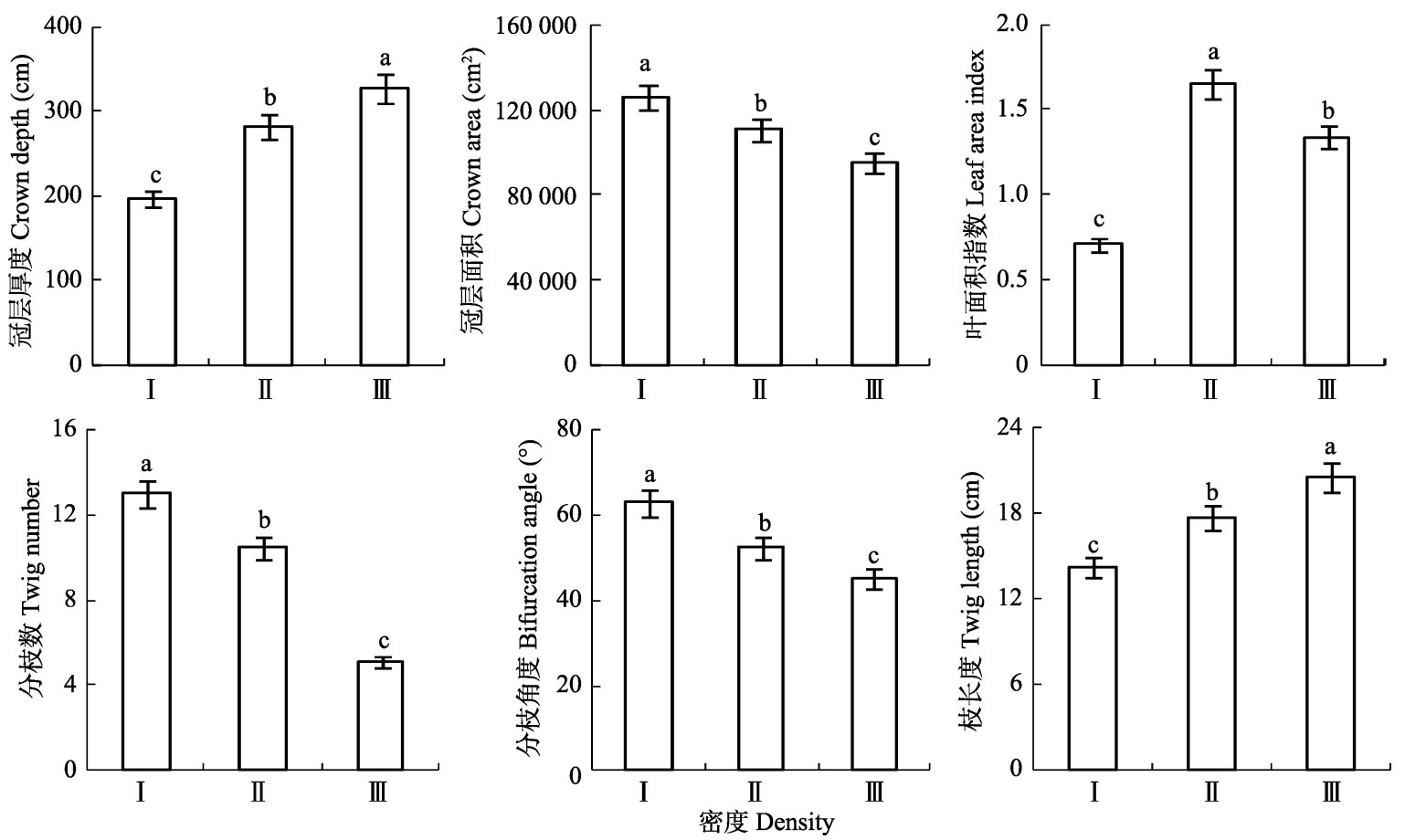

图1 不同密度条件下旱柳种群冠层构型的变化(平均值士标准误差)。不同小写字母表示在密度间差异显著 $(p<0.05)$ I、II、 III, 同表1。

Fig. 1 Canopy structure of Salix matsudana by density (mean $\pm S E$ ). The lowercase letters indicate significant differences among density $(p<0.05)$. I, II, III, see Table 1.

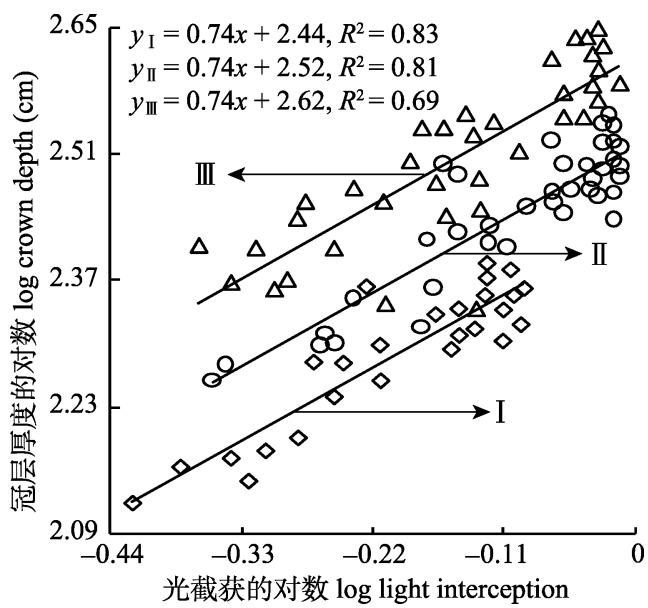

图2 不同密度旱柳光截获与冠层厚度的关系。I、II、III，同 表1。

Fig. 2 Relationship between light interception and crown depth of Salix matsudana by density. I, II, III, see Table 1.

SMA斜率均与-1存在极显著差异 $(p<0.01)$, 中密度 与1存在极显著差异 $(p<0.01)$, 表明旱柳在低密度和 高密度时, $L I$ 越大、冠层面积越小, 不同密度生境中 呈不同的权衡关系; 在中密度时, 旱柳LI越大、冠层 面积越大, $L I$ 增加的速度大于冠层面积增加的速度。

\section{3 讨论}

在森林群落中, 光分布与植株冠层的株高、叶

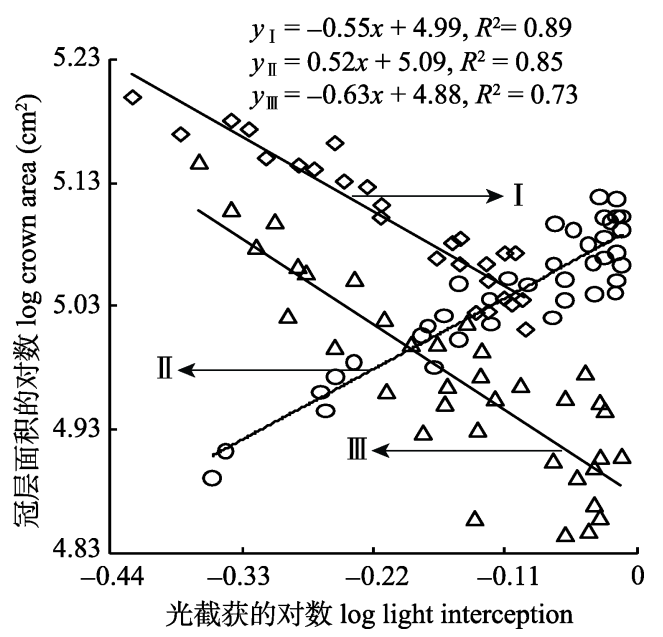

图3 不同密度旱柳冠层光截获与冠层面积的关系。I、II、 III, 同表1。

Fig. 3 Relationship between light interception and crown area of Salix matsudana by density. I, II, III, see Table 1.

面积指数以及叶片对光的吸收等关系密切，而这些 特征会因群体密度的升高而出现差异(郭江等, 2005), 造成异质性光环境, 从而对植物冠层构型产 生显著影响。本研究发现, 不同密度条件下土壤水 分差异不显著 $(p>0.05)$, 对湿地旱柳种群没有影响; 旱柳光截获与冠层厚度和冠层面积的关系与杨晓东 等(2013)对于“冠幅曝光指数与冠层厚度和冠层面 
积均呈显著正相关”的研究结果部分一致(图2, 图 3)。湿地环境中, 不同种群密度旱柳光截获和冠层 厚度、冠层面积关系的差异性, 既是密度制约对冠 层光辐射截取能力的塑造, 也是湿地植物根据自身 的生存需求, 在最佳资源配置模式下选择的光环境 适应机制。

\section{1 低密度条件下旱柳光截获与冠层厚度和冠层 面积的关系}

密度差异形成各种不同的光环境, 引起植株冠 层结构的动态变化, 使得植株叶片和枝条的形态 (Stenberg et al., 1998)和树冠形态(King, 1997)等都 表现出高度的可塑性。本研究中, 低密度样地旱柳 光截获与冠层厚度和面积分别呈极显著正相关和负 相关, 形成小冠层厚度、大冠层面积的冠层构型(图 1)，这符合段劼等(2010)提出的“林分密度较低时树 冠水平表面积大、生长饱满”的观点。主要原因可能 在于: (1)低密度种群的光、热和空间资源充足, 种群 竞争较弱, 旱柳为了拓展生存生态位, 优先倾向于 增加枝条数和分枝角度, 从而选择了减少枝长度和 单位分枝上叶面积的资源投资, 促使枝条水平伸 展、叶片松散排列在不同的分枝上表现为LAI很低 (王俊峰和冯玉龙, 2004), 较小的 $L A I$ 决定了植物冠 层较少的光截获量(图1)(Depury \& Farquhar, 1997); (2)邻体遮挡较少且 $P A R$ 最大(表2), 常常遭受强光胁 迫, 冠层内部枝条之间的自我荫蔽程度主要与植株 冠层形态特征有关, 冠层越深、幅度越小的植株个 体, 其自我荫蔽程度则越弱, 反之亦然(Osunkoya et al., 2007), 为了躲避强光胁迫, 植物选择增大胸 径和冠幅而降低株高与冠幅深度, 这是早柳在强光 下的自我保护构造和安全性经济权衡策略; (3)结合 表1、表 2 和图 1 资料认为, 低密度生境地表盐分积累 尤为明显, 电导率最大, 盐分胁迫造成的“生理干 旱”现象阻碍了植物的吸水作用(周洪华和李卫红, 2015), 使植物的生长高度和冠幅厚度都受到限制。 随密度改变, 旱柳净光合速率的下降幅度小于蒸腾 速率, 较高的水分利用率有利于旱柳将更多的光合 产物投入到与木质部水分、养分输导有关的系统构 建方面(史元春等, 2015), 横向扩展冠幅、加粗树干 以维持机械支持与导管畅通, 为水分转移至树顶提 供更大的接触面积和便捷的路径。因此, 旱柳在低 密度时, 光和盐胁迫加剧, 促使植物趋向于矮状化, 采取减少光截获的水平空间拓展模式来应对环境
胁迫。

\section{2 高密度条件下旱柳光截获与冠层厚度和冠层 面积的关系}

密度制约通过改变种群内每个植株可获得性 资源的数量, 使邻体间发生地上的光竞争和地下的 水分与矿质营养竞争(黎磊等, 2011)。本研究中, 早 柳在高密度光截获与冠幅厚度和面积分别呈显著正 相关和负相关, 形成大冠层厚度、小冠层面积的冠 层构型(图1), 这与段劼等(2010)关于“在高密度条 件下狭长型树冠能够获得更多的光照”的研究结果 一致。主要原因在于: (1)高密度种群郁闭度和拥挤 程度最大(表1), 来自邻株植物的遮阴降低了冠层内 部红外/远红外光比率(R/FR), 限制了可获得性光合 有效辐射(表2), 旱柳作出增加枝长度、减少分枝数 的遮阴躲避反应(图1), 将大量叶片聚集在树冠上部, 造成较高的 $L A I$, 有利于旱柳采取顶端优势策略进 行光资源竞争, 截获更多光辐射, 因而光照越弱, 植株的顶端优势越强, 植株冠层多呈窄的塔形结构 (Cline, 1991)；(2)密植条件下林分内剩余空间相对 降低, 种群内部个体间的竞争加剧, 植株通过减小 分枝角度，增大株高，促使枝条排列趋向于增加植 株相对冠层深度、减小冠幅, 这样既能减少在茎上 的投资消耗(因为依赖增大冠幅提高叶面积要求较 高的茎比例), 又能降低冠层内部的自我荫蔽程度 (Osunkoya et al., 2007); (3)该生境土壤电导率较低, 植物体内的水分含量充足, 植物降低WUE以较大蒸 腾速率进行光合碳的积累和分配，在枝叶大小投资 预算中，将单位物质投资偏向于叶片大小的扩展， 而牺牲支撑枝条大小扩展以适应水分充足的环境 (章建红等, 2014), 在冠层构型上表现出牺牲胸径和 冠幅大小而将更多的资源用于株高和冠层厚度的构 建。因此, 空间受限和弱光胁迫成为高密度旱柳生 长的限制因子, 为了保证有限空间下光合碳获取能 力最大化, 需要增大冠层厚度和减小冠层面积, 通 过垂直方向上拓展生存空间来提高光截获效率。

\section{3 中密度条件下旱柳光截获与冠层厚度和冠层 面积的关系}

植物形态、器官间的生长比率和郁闭度都受限 制因子和密度的影响(Zhang et al., 2008), 在特定的 环境下, 植物为了满足生存需求会形成不同的形态 结构, 使植物更好地调整和适应异质性资源(龚容 和高琼, 2015)。本研究中, 中密度样地旱柳光截获 
与冠层厚度和面积均形成了极显著正相关关系, 表 明光截获和冠层厚度的协调性比低高密度更强, 光 截获和冠层面积的关系则与低高密度相反, 反映了 中密度旱柳冠层能够充分调控冠层构型, 有效地截 获光能, 密度过大或过小均会造成光合效率下降, 冠层结构不良(张旺锋等, 2004)。主要原因可能有: (1)中密度样地旱柳的分枝角度、枝长度和分枝数适 中, 使得冠层内叶分布和光分布较为分散均匀, $P A R$ 在冠层中的均匀分布很重要(Kanniah et al., 2012), 可促进枝叶的合理配置与冠层形态的均衡发展, 有 利于光、热和空间资源的更合理利用和分配, 使植 物在竞争中占据优势, 达到更大限度地占有地上部 分资源和拓展空间的目的, 从而维持种群稳定性; (2)中密度条件下郁闭度中等, 有适度的遮阴现象, 降低了强光对植株的直接辐射, 有效地保证了电子 传递和能量利用, 增加了光截获效率, 并且旱柳种 群在中密度具有最高的 $L A I$ 也增大了冠层截取与吸 收太阳辐射的能力, 能够满足冠层在水平和垂直方 向同时拓展生存空间所需的光能消耗; (3)一定浓度 的土壤盐分对植物的生长发育有促进作用, 只有过 量的盐才对植物产生危害(孙洪刚和陈益泰, 2010), 旱柳是稍耐盐碱的树种, 中密度样地的土壤电导率 较低, 植物的水分利用效率被促进, 能够高效地将 光合同化产物均匀分配到地上部分的各个器官, 使 光截获与冠层构型更加契合。因此, 中密度样地适 宜的光照条件和土壤盐分浓度, 使其有效地进行光 合作用和水分运输, 旱柳选择同时拓展冠层厚度与 冠层面积最大限度提高光截获能力, 这对于种群的 稳定与扩张都是有利的。

\section{4 结论}

旱柳在低密度趋向于构建较小的冠层厚度、较 大的冠层面积来适合于较低的光截取能力, 采取水 平拓展的空间利用策略, 以应对强光、水分胁迫和 保持自身竞争优势; 高密度旱柳选择构建较大冠层 厚度与较小冠层面积来增大光截取能力, 通过空间 垂直拓展模式, 达到充分利用冠层顶部的光资源以 规避弱光胁迫的目的; 中密度旱柳则利用其光照资 源优势, 采取以提高资源利用效率为主的空间均衡 发展策略, 最大限度地发挥植物光合生理作用, 具 有较高的资源获取能力和匀称的冠层轮廓。不同密 度种群所选择的空间生存模式均体现了重要的碳-
水投资器官如何有效地调节资源分配，即光合能力 和水分运输能力的匹配问题。这种结果较好地验证 了植物通过冠层结构的可塑性调整响应环境变异的 协同适应性机制，这也说明旱柳作为洪泛平原湿地 的主要树种具有很强的环境适应性。本文仅从密度 角度分析了三者间的变化特征，它们的关系可能还 受到微地形因子、物种遗传特性以及林下植被生物 多样性和碳汇等因素的影响, 需要进一步的试验 验证。

\section{基金项目 国家自然科学基金 (41461013 和} 91125014)。

\section{参考文献}

Clark JS (2010). Individuals and the variation needed for high species diversity in forest trees. Science, 327, 1129-1132.

Cline MG (1991). Apical dominance. The Botanical Review, 57, 318-358.

Depury DGG, Farquhar GD (1997). Simple scaling of photosynthesis from leaves to canopies without the errors of big-leaf models. Plant, Cell \& Environment, 20, 537-557.

Du J, Zhao CZ, Song QH, Shi YC, Wang JW, Chen J (2016). Plant size differences with twig and leaf traits of Zygophyllum xanthoxylum in the northern slope of Qilian Mountains, China. Chinese Journal of Plant Ecology, 40, 212-220. (in Chinese with English abstract) [杜晶, 赵成 章, 宋清华, 史元春, 王继伟, 陈静 (2016). 祁连山北 坡霸王枝-叶性状关系的个体大小差异. 植物生态学报, 40, 212-220.]

Duan J, Ma LY, Jia LM, Xu CY, Jia ZK, Che WR (2010). The density effect of Platycladus orientalis plantation in Beijing area. Acta Ecologica Sinica, 30, 3206-3214. (in Chinese with English abstract) [段劼, 马履一, 贾黎明, 徐程 扬, 贾忠奎, 车文瑞 (2010). 北京地区侧柏人工林密度 效应. 生态学报, 30, 3206-3214.]

Goel NS (1988). Models of vegetation canopy rellectance and their use in estimation of biophysical parameters from rellectance data. Remote Sening Reviews, 4, 1-221.

Gong R, Gao Q (2015). Research progress in the effects of leaf hydraulic characteristics on plant physiological functions. Chinese Journal of Plant Ecology, 39, 300-308. (in Chinese with English abstract) [龚容, 高琼 (2015). 叶片结 构的水力学特性对植物生理功能影响的研究进展. 植 物生态学报, 39, 300-308.]

Guo J, Xiao K, Guo XY, Zhang FL, Zhao CZ (2005). Review on maize canopy structure, light distributing and canopy photosynthesis. Journal of Maize Science, 13(2), 55-59. (in Chinese with English abstract) [郭江, 肖凯, 郭新宇, 张凤路, 赵春江 (2005). 玉米冠层结构、光分布和光合 作用研究综述. 玉米科学, 13(2), 55-59.] 
Han L, Zhao CZ, Xu T, Feng W, Duan BB, Zheng HL. Trade-off between leaf size and vein density of Achnatherum splendens in Zhangye wetland. Chinese Journal of Plant Ecology, 40, 788-797. (in Chinese with English abstract) [韩玲, 赵成章, 徐婷, 冯威, 段贝贝, 郑慧玲 (2016). 张掖湿地芨芨草叶大小和叶脉密度的权衡关系. 植物生态学报, 40, 788-797.]

Japhet W, Zhou DW, Zhang HX, Zhang HX, Yu T (2009). Evidence of phenotypic plasticity in the response of $\mathrm{Fa}$ gopyrum esculentum to population density and sowing date. Journal of Plant Biology, 52, 303-311.

Kanniah KD, Beringer J, North P, Hutley L (2012). Control of atmospheric particles on diffuse radiation and terrestrial plant productivity: A review. Progress in Physical Geography, 36, 209-237.

Kind DA, Davies SJ, Noor NSM (2006). Growth and mortality are related to adult tree size in a Malaysian mixed dipterocarp forest. Forest Ecology and Management, 223, 152158.

King DA (1997). Branch growth and biomass allocation in Abies amabilis saplings in contrasting light environments. Tree Physiology, 17, 251-258.

Li L, Zhou DW, Sheng LX (2011). Density dependencedetermined plant biomass allocation pattern. Chinese Journal of Ecology, 30, 1579-1589. (in Chinese with English abstract) [黎磊, 周道玮, 盛连喜 (2011). 密度 制约决定的植物生物量分配格局. 生态学杂志, 30 , 1579-1589.]

Li SY, Feng W, Wang YH, Wang CY, Guo TC (2013). Effects of spacing interval of wide bed planting on canopy characteristics and yield in winter wheat. Chinese Journal of Plant Ecology, 37, 758-767. (in Chinese with English abstract) [李世荣, 冯伟, 王永华, 王晨阳, 郭天财 (2013). 宽幅播种带间距对冬小麦冠层特征及产量的影响. 植 物生态学报, 37, 758-767.]

Ma ZQ, Liu QJ, Zeng HQ, Li XR, Chen YR, Zhang SH, Yang FD, Wang HQ (2008). Estimation of leaf area index of planted forests in subtropical China by photogrammetry. Acta Ecologica Sinica, 28, 1971-1980. (in Chinese with English abstract) [马泽清, 刘琪憬, 曾慧卿, 李轩然, 陈 永瑞, 林耀明, 张时煌, 杨风亭, 汪宏清 (2008). 南方 人工林叶面积指数的摄影测量. 生态学报, 28, 19711980.]

Osunkoya OO, Omar-Ali K, Amit N, Dayan J, Daud DS, Sheng TK (2007). Comparative height crown allometry and mechanical design in 22 tree species of Kuala Belalong rainforest, Brunei, Borneo. American Journal of Botany, 94, 1951-1962.

Poorter L, Bongers F, Sterck FJ, Woll H (2003). Architecture of 53 rain forest tree species differing in adult stature and shade tolerance. Ecology, 84, 602-608.

Poorter L, Wright SJ, Paz H, Ackerly DD, Condit R, Ibarra-
Manriquez G, Harms KE, Licona JC, Martínez-Ramos M, Mazer SJ, Muller-Landau HC, Peña-Claros M, Webb CO, Wright IJ (2008). Are functional traits good predictors of demographic rates? Evidence from five neotropical forests. Ecology, 89, 1908-1920.

Rodríguez-González PM, Stella JC, Campelo F, Ferreira MT Albuquerque A (2010). Subsidy or stress? Tree structure and growth in wetland forests along a hydrological gradient in Southern Europe. Forest Ecology \& Management, 259, 2015-2025.

Shi YC, Zhao CZ, Song QH, Du J, Wang JW (2015). Allometric relationship between height and crown width or diameter of Platycladus orienalis on different slope aspects of Lanzhou northern mountains. Chinese Journal of Ecology, 34, 1879-1885. (in Chinese with English abstract) [史元 春, 赵成章, 宋清华, 杜晶, 王继伟 (2015). 兰州北山 侧柏株高与冠幅、胸径异速生长关系的坡向差异性. 生 态学杂志, 34, 1879-1885.]

Sixto H, Cañellas I, Arendonka JV, Ciria P, Camps F, Sánchez M, Sánchez-González M (2015). Growth potential of different species and genotypes for biomass production in short rotation in Mediterranean environments. Forest Ecology and Management, 354, 291-299.

Song X, Fang J, Han X, He X, Liu M, Hu J, Zhuo R (2016). Overexpression of quinone reductase from Salix matsudana Koidz enhances salt tolerance in transgenic Arabidopsis thaliana. Gene, 576, 520-527.

Stenberg P, Smolander H, Sprugel D, Smolander S (1998). Shoot structure, light interception, and distribution of nitrogen in an Abies amabilis canopy. Tree Physiology, 18, 759-767.

Sun HG, Chen YT (2010). Root growth patterns of four coastal shelter forest tree species in response to salt stress. Chinese Journal of Ecology, 29, 2365-2372. (in Chinese with English abstract) [孙洪刚, 陈益泰 (2010). 沿海防护林 四个树种根系分布对盐胁迫的响应. 生态学杂志, 29 , 2365-2372.]

Thorpe HC, Astrup R, Trowbridge A, Coates KD (2010). Competition and tree crowns: A neighborhood analysis of three boreal tree species. Forest Ecology and Management, 259, 1586-1596.

Wang JF, Feng YL (2004). The effect of light intensity on blomass allocation, leaf morphology and relative growth rate of two invasive plants. Acta Phytoecologica Sinica, 28, 781-786. (in Chinese with English abstract) [王俊峰, 冯 玉龙 (2004). 光强对两种入侵植物生物量分配、叶片形 态和相对生长速率的影响. 植物生态学报, 28, 781786.]

Warton DI, Wright IJ, Falster DS, Westoby M (2006). Bivariate line-fitting methods for allometry. Biological Reviews, 81, 259-291.

Wunsche JN, Lakso AN, Robinson TL, Lenz F, Denning SS

www.plant-ecology.com 
(1996). The bases of productivity in apple production systems: The role of light interception by different shoot types. Journal of the American Society for Horticultural Science, 121, 886-893.

Xia JB, Zhang SY, Zhao ZG, Zhao YY, Gao Y, Gu GY, Sun JK (2013). Critical effect of photosynthetic efficiency in Salix matsudana to soil moisture and its threshold grade in shell ridgei sland. Chinese Journal of Plant Ecology, 37, 851-860. (in Chinese with English abstract) [夏江宝, 张 淑勇, 赵自国, 赵艳云, 高源, 谷广义, 孙景宽 (2013). 贝壳堤岛旱柳光合效率的土壤水分临界效应及其阈值 分级. 植物生态学报, 37, 851-860.]

Yang XD, Yan ER, Zhang ZH, Sun BW, Huang HX, ARSHAD A, Ma WJ, Shi QR (2013). Tree architecture of overlapping species among successional stages in evergreen broad-leaved forests in Tiantong region, Zhejiang Province, China. Chinese Journal of Plant Ecology, 37, 611-619. (in Chinese with English abstract) [杨晓东, 阎 恩荣, 张志浩, 孙宝伟, 黄海侠, ARSHAD A, 马文济, 史青茹 (2013). 浙江天童常绿阔叶林演替阶段共有种 的树木构型. 植物生态学报, 37, 611-619.]

Zhang H, Li JN, Wang GX, Dai XF, Qiu MQ, Zheng KF. (2008). Plants interactions between Suaeda salsa individuals are mediated by salinity stress. Acta Physiologiae Plantarum, 30, 99-104.

Zhang JH, Shi QR, Xu MS, Zhao YT, Zhong Q, Zhang FJ, Yan ER (2014). Testing of Corner's rules across woody plants in Tiantong region, Zhejiang Province: Effects of individ- ual density. Chinese Journal of Plant Ecology, 38, 655-664. (in Chinese with English abstract) [章建红, 史 青茹, 许洺山, 赵延涛, 仲强, 张富杰, 阎恩荣 (2014). 浙江天童木本植物Corner法则的检验: 个体密度的影 响. 植物生态学报, 38, 655-664.]

Zhang WF, Wang ZL, Yu SL, Li SK, Fang J, Tong WS (2004). Effects of planting density on canopy photosynthesis, canopy structures and yield formation of high-yield cotton in Xinjiang, China. Acta Phytoecologica Sinica, 28, 164-171. (in Chinese with English abstract) [张旺锋, 王 振林, 余松烈, 李少昆, 房建, 童文崧 (2004). 种植密 度对新疆高产棉花群体光合作用、冠层结构及产量形成 的影响. 植物生态学报, 28, 164-171.]

Zhang YQ, Liang CZ, Wang W, Wang LX, Peng JT, Yan JC, Jia JC (2010). Soil salinity and Achnatherum splendens distribution. Chinese Journal of Ecology, 29, 2438-2443. (in English with Chinese abstract) [张雅琼, 梁存柱, 王 炜, 王立新, 彭江涛, 间建成, 贾成朕 (2010). 芨苃草 群落土壤盐分特征. 生态学杂志, 29, 2438-2443.]

Zhou HH, Li WH (2015). Responses and adaptation of xylem hydraulic conductivity to salt stress in Populus euphratica. Chinese Journal of Plant Ecology, 39, 81-91 (in English with Chinese abstract) [周洪华, 李卫红 (2015). 胡杨木 质部水分传导对盐胁迫的响应与适应. 植物生态学报, 39, 81-91.]

责任编委: 孙书存 责任编辑: 李 敏

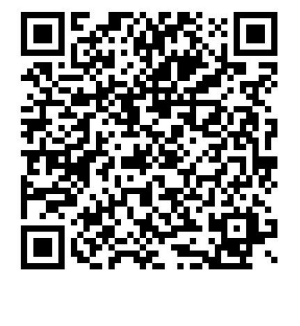

